Vietnam Journal of Mechanics, VAST, Vol.40, No.3 (2018), pp. 285-301

DOI: https://doi.org/10.15625/0866-7136/12397

\title{
NONLINEAR BUCKLING OF CNT-REINFORCED COMPOSITE TOROIDAL SHELL SEGMENT SURROUNDED BY AN ELASTIC MEDIUM AND SUBJECTED TO UNIFORM EXTERNAL PRESSURE
}

\author{
Hoang Van Tung ${ }^{1, *}$, Pham Thanh Hieu ${ }^{2}$ \\ ${ }^{1}$ Hanoi Architectural University, Vietnam \\ ${ }^{2}$ University of Transport Technology, Hanoi, Vietnam \\ *E-mail: hoangtung0105@gmail.com \\ Received April 24, 2018
}

\begin{abstract}
Buckling and postbuckling behaviors of Toroidal Shell Segment (TSS) reinforced by single-walled carbon nanotubes (SWCNT), surrounded by an elastic medium and subjected to uniform external pressure are investigated in this paper. Carbon nanotubes (CNTs) are reinforced into matrix phase by uniform distribution (UD) or functionally graded (FG) distribution along the thickness direction. Effective properties of carbon nanotube reinforced composite (CNTRC) are estimated by an extended rule of mixture through a micromechanical model. Governing equations for TSSs are based on the classical thin shell theory taking into account geometrical nonlinearity and surrounding elastic medium. Three-term solution of deflection and stress function are assumed to satisfy simply supported boundary condition, and Galerkin method is applied to obtain nonlinear load-deflection relation from which buckling loads and postbuckling equilibrium paths are determined. The effects of CNT volume fraction, distribution types, geometrical ratios and elastic foundation on the buckling and postbuckling behaviors of CNTRC TSSs are analyzed and discussed.
\end{abstract}

Keywords: CNT-reinforced composite, toroidal shell segment, buckling and postbuckling, mechanical load, elastic foundation.

\section{INTRODUCTION}

Since their authenticated introduction, nearly three decades ago, carbon nanotubes (CNTs), a new class of advanced materials, have attracted huge attention of researchers. Single walled carbon nanotube (SWCNT) consists of a single sheet of graphene rolled seamlessly to form a cylinder having diameter of order of $1 \mathrm{~nm}$ and length of up to centimeters. The researches of material scientists shown that CNTs have extremely high elastic modulus, greater than $1 \mathrm{TPa}$ and strengths 10-100 times higher than the strongest steel at a fraction of the weight $[1,2]$. In addition to these extraordinary mechanical properties

(C) 2018 Vietnam Academy of Science and Technology 
associated with CNTs, they also posses superior thermal and electrical properties. Due to unprecedentedly excellent characteristics, CNTs are used as ideal fillers into isotropic matrix phase to form nanocomposite known as carbon nanotube reinforced composite (CNTRC). The CNT-reinforced composites can be fabricated by solution processing of CNTs and polymer, bulk mixing, melt mixing or in situ polymerization [3]. Based on idea of functionally graded material (FGM), Shen [4] proposed the concept of functionally graded carbon nanotube reinforced composite (FG-CNTRC) properties of which are graded in the thickness direction according to functional rules to obtain optimal distribution of CNTs and the desired response of CNTRC structures. Stimulated by Shen's proposal, investigations on static and dynamic responses of FG-CNTRC plates and shells have been performed.

Linear static stability of FG-CNTRC rectangular and skew plates under mechanical loads have been treated in works of Liew and co-workers $[5,6]$ using numerical methods. Linear mechanical buckling and free vibration of FG-CNTRC plates have been examined in works [7] and [8] employing semi-analytical and analytical solutions, respectively. Buckling behavior of FG-CNTRC rectangular and skew plates under uniform temperature rise has been investigated by Mirzaei and Kiani [9] and Kiani [10] using Ritz method. Employing first order shear deformation theory and differential quadrature methods, mechanical buckling behavior of quadrilateral laminated plates composed of perfectly bonded CNTRC layers with arbitrary boundary conditions has been treated in works of Malekzadeh and Shojaee [11], Setoodeh and Shojaee [12]. Based on a two-step perturbation technique, Shen and Zhang [13] studied the postbuckling behavior of FG-CNTRC plates under thermal loads. Using Ritz method with Chebyshev polynomials as shape functions, Kiani [14] dealt with thermal postbuckling problems of FG-CNTRC plates with various boundary conditions. Zhang and Liew [15] used a mesh-free method to analyze postbuckling behavior of axially compressed FG-CNTRC plates on elastic foundations. Recently, the effects of tangential edge constraints on the nonlinear stability of thin FGCNTRC plates on elastic foundation under thermal, mechanical and thermomechanical loads have been analyzed in works of Tung [16] and Trang and Tung [17].

Linear and nonlinear stability of FG-CNTRC cylindrical panels have been addressed in works [18-23]. Nasihatgozar et al. [18] employed the analytical solution to study linear buckling of FG-CNTRC cylindrical panels with piezoelectric layer under axial compression. Basing on a numerical approach with shell finite element, Macias et al. [19] analyzed linear buckling of FG-CNTRC cylindrical panels under axial compression and shear. Using asymptotic solutions and a perturbation technique, Shen and Xiang [20] and Shen [21] presented results for postbuckling analysis of FG-CNTRC cylindrical panels subjected to axial compression and external pressure in thermal environments, respectively. Very recently, Trang and Tung $[22,23]$ used an analytical approach to investigate effects of tangential constraints of boundary edges on the nonlinear stability of thin FG-CNTRC cylindrical panels under axial compression and external pressure.

Based on a semi-analytical approach with asymptotic solutions and a perturbation technique, buckling and postbuckling behaviors of FG-CNTRC circular cylindrical shells subjected to axial compression and external pressure loads in thermal environments have been analyzed by Shen $[24,25]$ employing a higher order shear deformation theory. Shen 
$[26,27]$ also analyzed the postbuckling behavior of FG-CNTRC cylindrical shells under thermal and torsional loadings. Making use of analytical approaches with three-term solution of deflection and classical thin shell theory, Ansari et al. [28] and Ninh [29] considered the postbuckling behavior of FG-CNTRC cylindrical shells with piezoelectric layers subjected to external pressure and torsional loads, respectively. Although postbuckling behavior of ceramic-metal FGM toroidal shell segments has been addressed in works [30,31], to the best of authors' knowledge, there is no investigation on nonlinear stability of CNTRC toroidal shell segments. As important structural components, CNTRC toroidal shell segments have potential applications in many different scientific fields, especially in aerospace science and engineering [32].

This paper presents an analytical investigation on the buckling and postbuckling behaviors of composite toroidal shell segments reinforced by single-walled carbon nanotubes, surrounded by an elastic medium and subjected to uniform external pressure. Carbon nanotubes are embedded into matrix phase by uniform distribution (UD) or four types of functionally graded (FG) distribution. Effective properties of carbon nanotube reinforced composite (CNTRC) are estimated by an extended rule of mixture through a micromechanical model. Formulations are based on the classical shell theory taking geometrical nonlinearity and surrounding medium-shell interaction into consideration. Three-term solution of deflection and stress function are assumed to satisfy simply supported boundary conditions, and Galerkin method is applied to obtain nonlinear loaddeflection relation from which buckling pressures and postbuckling curves are determined. Numerical examples are carried out to analyze the effects of CNT volume fraction, distribution types, geometrical ratios and elastic foundation on the buckling loads and postbuckling load carrying capacity of pressure-loaded CNTRC toroidal shell segments.

\section{CNT-REINFORCED COMPOSITE TOROIDAL SHELL SEGMENT (CNTRC TSS) SURROUNDED BY AN ELASTIC MEDIUM}

Consider a toroidal shell segment of thickness $h$ and length $L$ formed by rotation of a plane circular arc about an axis in the plane of the arc. The principal radii of curvature of the middle surface of the shell segment in the meridian and circumferential directions are denoted by $a$ and $R$, respectively. The toroidal shell segment is surrounded by an elastic medium modelled as a two-parameter elastic foundation and defined in a coordinate system $x y z$ origin of which is located on the middle surface at an end, $x$ and $y$ are in meridian and circumferential directions, respectively, and $z$ is perpendicular to the middle surface as shown in Fig. 1.

For the middle surface of a segment of a toroidal shell, from Fig. 1, $r=R-a(1-$ $\sin \varphi)$ in which $\varphi$ denotes the angle between the axis of revolution and a normal to the middle surface of shell segment. For a sufficiently shallow segment in the region of the equator of the torus, the angle $\varphi$ is approximately equal to $\pi / 2$ and, as a result, $\sin \varphi \approx 1$, $\cos \varphi \approx 0$, and $r \approx R$. Then, the form of the equations mentioned later is simplified by setting $\mathrm{d} x=a \mathrm{~d} \varphi$ and $\mathrm{d} y=\operatorname{Rd} \theta$ where $\theta$ is a circumferential coordinate of a point on the middle surface. It is noted that the radius $a$ is positive and negative with convex and concave TSS, respectively. 


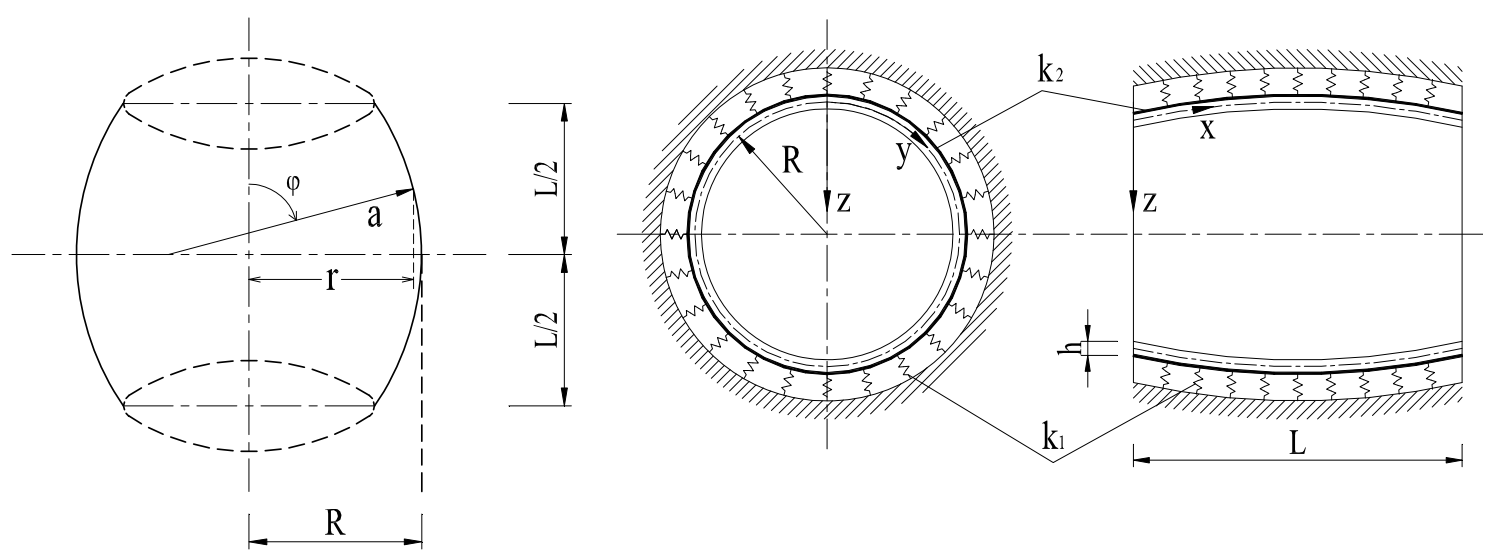

Fig. 1. Configuration and coordinate system of a toroidal shell segment (TSS) surrounded by an elastic medium

In this study, SWCNTs are reinforced into isotropic polymer matrix by uniform distribution (UD) or four functionally graded (FG) distributions named as FG-V, FG- $\Lambda$, FG$\mathrm{O}$ and FG-X types. Specifically, the outer and inner surfaces of the TSS are CNT-rich for FG-V and FG- $\Lambda$ types, respectively. The middle surface of the TSS is CNT-rich in case of FG-O type and both outer and inner surfaces are enriched by CNTs for FG-X type. According to a micromechanical model, effective properties of composite materials may be estimated based on the Mori-Tanaka approach or the rule of mixture. It is known that rule of mixture is simple and efficiently applied to predict overall properties of composites, and an excellent agreement between Mori-Tanaka approach and rule of mixture for buckling analysis results of FG-CNTRC shell panels has been proven in the work [19]. In the present work, extended rule of mixture is applied to determine effective Young moduli and shear modulus of the CNTRC TSS as [4]

$$
\begin{gathered}
E_{11}=\eta_{1} V_{C N T} E_{11}^{C N T}+V_{m} E^{m}, \\
\frac{\eta_{2}}{E_{22}}=\frac{V_{C N T}}{E_{22}^{C N T}}+\frac{V_{m}}{E^{m}}, \\
\frac{\eta_{3}}{G_{12}}=\frac{V_{C N T}}{G_{12}^{C N T}}+\frac{V_{m}}{G^{m}},
\end{gathered}
$$

where $E_{11}^{C N T}, E_{22}^{C N T}$ and $G_{12}^{C N T}$ are the Young moduli and shear modulus, respectively, of the CNT, whereas $E^{m}$ and $G^{m}$ are Young and shear moduli, respectively, of the isotropic matrix. Coefficients $\eta_{j}(j=1,2,3)$, called the CNT efficiency parameters, are introduced into Eqs. (1) to consider the size-dependent material properties, whereas $V_{C N T}$ and $V_{m}$ are the volume fractions of CNTs and matrix, respectively, and their relation is $V_{C N T}+V_{m}=1$. 
The volume fractions $V_{C N T}$ for five types of CNT distributions are assumed as

$$
V_{C N T}= \begin{cases}V_{C N T}^{*} & (\mathrm{UD}) \\ \left(1+\frac{2 z}{h}\right) V_{C N T}^{*} & (\mathrm{FG}-\Lambda) \\ \left(1-\frac{2 z}{h}\right) V_{C N T}^{*} & (\mathrm{FG}-\mathrm{V}) \\ 2\left(1-\frac{2|z|}{h}\right) V_{C N T}^{*} & (\mathrm{FG}-\mathrm{O}) \\ 2\left(\frac{2|z|}{h}\right) V_{C N T}^{*} & (\mathrm{FG}-\mathrm{X})\end{cases}
$$

where

$$
V_{C N T}^{*}=\frac{w_{C N T}}{w_{C N T}+\left(\rho_{C N T} / \rho_{m}\right)\left(1-w_{C N T}\right)},
$$

in which $w_{C N T}$ is the mass fraction of CNTs in the CNTRC TSS, $\rho_{C N T}$ and $\rho_{m}$ are the densities of the CNTs and matrix, respectively. Poisson ratio is determined by

$$
v_{12}=V_{C N T}^{*} v_{12}^{C N T}+V_{m} v^{m},
$$

where $v_{12}^{C N T}$ and $v^{m}$ are Poisson ratios of the CNT and matrix, respectively.

\section{FORMULATIONS}

Based on the classical shell theory (CST), strain components are expressed as

$$
\varepsilon_{x}=\varepsilon_{x 0}+z \chi_{x}, \quad \varepsilon_{y}=\varepsilon_{y 0}+z \chi_{y}, \quad \gamma_{x y}=\gamma_{x y 0}+2 z \chi_{x y},
$$

where strains of middle surface $\varepsilon_{x 0}, \varepsilon_{y 0}, \gamma_{x y 0}$ and curvature changes $\chi_{x}, \chi_{y}, \chi_{x y}$ are related to displacements of middle surface $u, v, w$ in coordinate directions $x, y, z$, respectively, as follows

$$
\left(\begin{array}{c}
\varepsilon_{x 0} \\
\varepsilon_{y 0} \\
\gamma_{x y 0}
\end{array}\right)=\left(\begin{array}{c}
u_{, x}-w / a+w_{, x}^{2} / 2 \\
v_{, y}-w / R+w_{, y}^{2} / 2 \\
u_{, y}+v_{, x}+w_{, x} w_{, y}
\end{array}\right), \quad\left(\begin{array}{c}
\chi_{x} \\
\chi_{y} \\
\chi_{x y}
\end{array}\right)=\left(\begin{array}{c}
-w_{, x x} \\
-w_{, y y} \\
-w_{, x y}
\end{array}\right),
$$

where subscript prime indicates partial derivative and von Karman-Donnell nonlinear terms are maintained. Based on Hooke's law, stress components in CNTRC TSS are expressed as

$$
\left(\begin{array}{c}
\sigma_{x} \\
\sigma_{y} \\
\sigma_{x y}
\end{array}\right)=\left(\begin{array}{ccc}
Q_{11} & Q_{12} & 0 \\
Q_{12} & Q_{22} & 0 \\
0 & 0 & Q_{66}
\end{array}\right)\left(\begin{array}{c}
\varepsilon_{x} \\
\varepsilon_{y} \\
\gamma_{x y}
\end{array}\right)
$$

where

$$
Q_{11}=\frac{E_{11}}{1-v_{12} v_{21}}, \quad Q_{22}=\frac{E_{22}}{1-v_{12} v_{21}}, \quad Q_{12}=\frac{v_{21} E_{11}}{1-v_{12} v_{21}}, \quad Q_{66}=G_{12} .
$$


Force and moment resultants in the CNTRC TSS are expressed as

$$
\begin{aligned}
& \left(N_{x}, M_{x}\right)=\left(e_{11}, e_{12}\right) \varepsilon_{x 0}+v_{21}\left(e_{11}, e_{12}\right) \varepsilon_{y 0}+\left(e_{12}, e_{13}\right) \chi_{x}+v_{21}\left(e_{12}, e_{13}\right) \chi_{y}, \\
& \left(N_{y}, M_{y}\right)=v_{12}\left(e_{21}, e_{22}\right) \varepsilon_{x 0}+\left(e_{21}, e_{22}\right) \varepsilon_{y 0}+v_{12}\left(e_{22}, e_{23}\right) \chi_{x}+\left(e_{22}, e_{23}\right) \chi_{y}, \\
& \left(N_{x y}, M_{x y}\right)=\left(e_{31}, e_{32}\right) \gamma_{x y 0}+2\left(e_{32}, e_{33}\right) \chi_{x y},
\end{aligned}
$$

where coefficients $e_{i j}(i, j=1 \div 3)$ are defined as in works [16,17,22,23].

Based on the CST, nonlinear equilibrium equations for a TSS surrounded by an elastic medium are [31]

$$
\begin{aligned}
& N_{x, x}+N_{x y, y}=0, \\
& N_{x y, x}+N_{y, y}=0, \\
M_{x, x x}+2 M_{x y, x y}+M_{y, y y}+N_{x} w_{, x x}+ & 2 N_{x y} w_{, x y}+N_{y} w_{, y y}+N_{x} / a+N_{y} / R+q-q_{f}=0,
\end{aligned}
$$

where $q$ is uniform external pressure and $q_{f}$ is interactive pressure between the TSS and surrounding medium and represented by Pasternak model as

$$
q_{f}=k_{1} w-k_{2} \Delta w,
$$

in which $k_{1}$ is Winkler foundation modulus, $k_{2}$ is stiffness of shear layer in Pasternak model and $\Delta=\partial^{2} / \partial x^{2}+\partial^{2} / \partial y^{2}$ is Laplace operator.

From Eqs. (6) and (9), equilibrium equation of a CNTRC TSS is rewritten in the form as

$$
\begin{array}{r}
a_{11} w_{, x x x x}+a_{21} w_{, y y y y}+a_{31} w_{, x x y y}+a_{41} f_{, x x y y}-f_{, y y} w_{, x x}+2 f_{, x y} w_{, x y} \\
-f_{, x x} w_{, y y}-\frac{f_{, y y}}{a}-\frac{f_{, x x}}{R}-q+k_{1} w-k_{2}\left(w_{, x x}+w_{, y y}\right)=0,
\end{array}
$$

where $f(x, y)$ is a stress function making Eqs. (10a) and (10b) identically satisfied according to the following definition

$$
N_{x}=f_{, y y}, \quad N_{y}=f_{, x x}, \quad N_{x y}=-f_{, x y},
$$

and coefficients $a_{11}, a_{21}, a_{31}, a_{41}$ are given in the works [16,17].

Next, strain compatibility equation of a TSS is defined as [31]

$$
\varepsilon_{x 0, y y}+\varepsilon_{y 0, x x}-\gamma_{x y 0, x y}=w_{, x y}^{2}-w_{, x x} w_{, y y}-w_{, y y} / a-w_{, x x} / R .
$$

By virtue of Eqs. (6), (9) and (13), the strain compatibility equation of a geometrically perfect CNTRC TSS has the form

$$
\begin{array}{r}
a_{12} f_{, x x x x}+a_{22} f_{, x x y y}+a_{32} f_{, y y y y}+a_{42} w_{, x x x x}+a_{52} w_{, x x y y}+a_{62} w_{, y y y y} \\
-w_{, x y}^{2}+w_{, x x} w_{, y y}+\frac{w_{, y y}}{a}+\frac{w_{, x x}}{R}=0,
\end{array}
$$

where coefficients $a_{i 2}(i=1 \div 6)$ have been defined in the works [16,17].

The present study considers simply supported CNTRC TSS with freely movable edges and the associated boundary conditions are [30,31,33]

$$
w=0, \quad M_{x}=0, \quad N_{x y}=0, \quad N_{x}=N_{x 0} \quad \text { at } \quad x=0, L
$$


where $N_{x 0}$ is prebuckling axial fore resultant and zero-valued in the present study. The following three-term solution of deflection and stress function are assumed to satisfy approximately boundary conditions (16) [31]

$$
\begin{gathered}
w(x, y)=W_{0}+W_{1} \sin \beta_{m} x \sin \delta_{n} y+W_{2} \sin ^{2} \beta_{m} x, \\
f(x, y)=A_{1} \cos 2 \beta_{m} x+A_{2} \cos 2 \delta_{n} y+A_{3} \sin \beta_{m} x \sin \delta_{n} y+A_{4} \sin 3 \beta_{m} x \sin \delta_{n} y-\sigma_{0 y} h \frac{x^{2}}{2},
\end{gathered}
$$

where $\beta_{m}=m \pi / L, \delta_{n}=n / R$ with $m, n$ are numbers of half wave and full wave in the axial and circumferential directions, respectively, $W_{0}, W_{1}$ and $W_{2}$ are unknown amplitudes corresponding to prebuckling, linear buckling and nonlinear buckling states of the deflection, respectively. In addition, in Eq. (18), $\sigma_{0 y}$ is average stress in circumferential direction and $A_{i}(i=1 \div 4)$ are coefficients to be determined. Next, introduction of solutions (17) and (18) into the compatibility equation (15) gives the coefficients $A_{i}(i=1 \div 4)$ as follows

$$
\begin{aligned}
& A_{1}=\frac{1}{32 a_{12} \beta_{m}^{2}}\left(\delta_{n}^{2} W_{1}^{2}+16 a_{42} \beta_{m}^{2} W_{2}-\frac{4}{R} W_{2}\right), \quad A_{2}=\frac{\beta_{m}^{2} W_{1}^{2}}{32 a_{32} \delta_{n}^{2}}, \\
& A_{3}=\frac{W_{1}}{a_{12} \beta_{m}^{4}+a_{22} \beta_{m}^{2} \delta_{n}^{2}+a_{32} \delta_{n}^{4}}\left(\frac{\beta_{m}^{2}}{R}+\frac{\delta_{n}^{2}}{a}-a_{42} \beta_{m}^{4}-a_{52} \beta_{m}^{2} \delta_{n}^{2}-a_{62} \delta_{n}^{4}-\beta_{m}^{2} \delta_{n}^{2} W_{2}\right), \\
& A_{4}=\frac{\beta_{m}^{2} \delta_{n}^{2} W_{1} W_{2}}{81 a_{12} \beta_{m}^{4}+9 a_{22} \beta_{m}^{2} \delta_{n}^{2}+a_{32} \delta_{n}^{4}} .
\end{aligned}
$$

Subsequently, solutions (17) and (18) are placed into the equilibrium equation (12), then applying Galerkin method on whole region of the TSS $(0 \leq x \leq L, 0 \leq y \leq 2 \pi R)$ to the resulting equilibrium equation yields the following expressions

$$
\begin{gathered}
\sigma_{0 y} \frac{h}{R}-q+k_{1}\left(W_{0}+\frac{W_{2}}{2}\right)=0, \\
a_{13}-a_{23} W_{2}+a_{33} W_{1}^{2}+a_{43} W_{2}^{2}-\sigma_{0 y} h \delta_{n}^{2}=0, \\
k_{1} W_{0}+a_{14} W_{2}-a_{24} W_{1}^{2}+a_{34} W_{1}^{2} W_{2}+\sigma_{0 y} \frac{h}{R}-q=0,
\end{gathered}
$$

where coefficients $a_{j 3}(j=1 \div 4)$ and $a_{k 4}(k=1 \div 3)$ are displayed in Eq. (A1) in Appen$\operatorname{dix}$ A.

For the TSS, the following circumferential closed condition must be satisfied [34]

$$
\int_{0}^{2 \pi R} \int_{0}^{L} \frac{\partial v}{\partial y} \mathrm{~d} x \mathrm{~d} y=0 .
$$

From Eqs. (6), (9) and (13), $v, y$ is expressed in terms of partial derivatives of $w$ and $f$. Then substituting the solutions (17), (18) into the $v, y$ and putting the obtained result into Eq. (21) yield the following relation

$$
\sigma_{0 y} h=\left(1-v_{12} v_{21}\right) e_{21}\left[\frac{1}{R}\left(W_{0}+\frac{W_{2}}{2}\right)-\frac{\delta_{n}^{2}}{8} W_{1}^{2}\right] .
$$



tions

Introduction of $\sigma_{0 y}$ from Eq. (22) into Eqs. (20) gives the following system of equa-

$$
\begin{gathered}
b_{11}\left(2 \bar{W}_{0}+\bar{W}_{2}\right)-b_{21} \bar{W}_{1}^{2}-q=0 \\
b_{12}-b_{22} \bar{W}_{0}-b_{32} \bar{W}_{2}+b_{42} \bar{W}_{1}^{2}+b_{52} \bar{W}_{2}^{2}=0 \\
b_{13} \bar{W}_{0}+b_{23} \bar{W}_{2}-b_{33} \bar{W}_{1}^{2}+b_{43} \bar{W}_{1}^{2} \bar{W}_{2}-q=0
\end{gathered}
$$

where

$$
\left(\bar{W}_{0}, \bar{W}_{1}, \bar{W}_{2}\right)=\frac{1}{h}\left(W_{0}, W_{1}, W_{2}\right),
$$

and the details of coefficients $b_{i 1}(i=1 \div 2), b_{j 2}(j=1 \div 5)$ and $b_{k 3}(k=1 \div 4)$ can be found in Eqs. (A2) and (A3) in Appendix A.

Solving Eqs. (23) and (24) for $\bar{W}_{0}$ and $\bar{W}_{1}^{2}$ yields

$$
\begin{aligned}
& \bar{W}_{0}=b_{14}+b_{24} \bar{W}_{2}+b_{34} \bar{W}_{2}^{2}-b_{44} q, \\
& \bar{W}_{1}^{2}=b_{15}+b_{25} \bar{W}_{2}+b_{35} \bar{W}_{2}^{2}-b_{45} q,
\end{aligned}
$$

where coefficients $b_{i 4}$ and $b_{i 5}(i=1 \div 4)$ are displayed in Eq. (B1) of Appendix B.

Now, putting $\bar{W}_{0}$ and $\bar{W}_{1}^{2}$ from Eqs. (27) into Eq. (25) gives the following nonlinear relation

$$
q=\frac{1}{\left(b_{16}+b_{26} \bar{W}_{2}\right)}\left(b_{36}+b_{46} \bar{W}_{2}+b_{56} \bar{W}_{2}^{2}+b_{66} \bar{W}_{2}^{3}\right)
$$

where coefficients $b_{i 6}(i=1 \div 6)$ can be found in Eq. (B2) of Appendix B.

By setting $\bar{W}_{2}=0$, buckling pressures of CNTRC TSSs are obtained as

$$
q_{b}=\frac{b_{36}}{b_{16}}
$$

and critical buckling pressure $q_{c r}$ is the smallest value among values of $q_{b}$ with respect to numbers $m, n$.

It is obvious from Eq. (17) that maximum deflection of the CNTRC TSSs is

$$
W_{\max }=W_{0}+W_{1}+W_{2}
$$

Combining Eqs. (27) and Eq. (30) leads to the following expression of non-dimensional maximum deflection

$$
\bar{W}_{\max }=W_{\max } / h=b_{14}+\left(b_{24}+1\right) \bar{W}_{2}+b_{34} \bar{W}_{2}^{2}-b_{44} q+\left(b_{15}+b_{25} \bar{W}_{2}+b_{35} \bar{W}_{2}^{2}-b_{45} q\right)^{1 / 2} \text {. }
$$

Eqs. (28) and (31) are used for postbuckling analysis of CNTRC TSSs under uniform external pressure. 


\section{RESULTS AND DISCUSSION}

This section presents numerical results for CNTRC TSSs made of Poly (methyl methacrylate), referred to as PMMA, as matrix material and reinforced by $(10,10)$ SWCNTs. The properties of the PMMA are $E^{m}=2.5 \mathrm{GPa}, v^{m}=0.34$ and those of the $(10,10)$ SWCNTs are $E_{11}^{C N T}=5.6466 \mathrm{TPa}, E_{22}^{C N T}=7.0800 \mathrm{TPa}, G_{12}^{C N T}=1.9445 \mathrm{TPa}$ and $v_{12}^{C N T}=0.175$ at room temperature $(300 \mathrm{~K})[13,24,35]$. The CNT efficiency parameters $\eta_{j}(j=1 \div 3)$ are estimated by matching the Young moduli $E_{11}, E_{22}$ and shear modulus $G_{12}$ of the CNTRC determined from the extended rule of mixture to those from the molecular dynamics (MD) simulations and given in the works $[13,24,26]$ as $\left(\eta_{1}, \eta_{2}, \eta_{3}\right)=$ $(0.137,1.022,0.715)$ for case of $V_{C N T}^{*}=0.12,\left(\eta_{1}, \eta_{2}, \eta_{3}\right)=(0.142,1.626,1.138)$ for case of $V_{C N T}^{*}=0.17$ and $\left(\eta_{1}, \eta_{2}, \eta_{3}\right)=(0.141,1.585,1.109)$ for case of $V_{C N T}^{*}=0.28$.

As reviewed above, there is no investigation on buckling of CNTRC TSSs in the literature. Therefore, the buckling behavior of pressure-loaded CNTRC cylindrical shell, a special case of CNTRC TSS as meridian radius tends to infinity $(a \rightarrow \infty)$, is considered to verify the present study. The critical buckling pressures of simply supported CNTRC cylindrical shells with movable edges and without elastic foundation are shown in Tab. 1 in comparison with Shen's results [25] using asymptotic solutions and a perturbation technique. It is evident that a good agreement is achieved in this comparison.

Table 1. Comparison of critical buckling loads $q_{c r}$ (in $\mathrm{kPa}$ ) of CNTRC cylindrical shells under uniform external pressure $\left(R / h=100, R / a=0, K_{1}=K_{2}=0\right)$

\begin{tabular}{|c|c|c|c|c|c|c|c|}
\hline \multirow{2}{*}{$L / R$} & \multirow{2}{*}{ Source } & \multicolumn{2}{|c|}{$V_{C N T}^{*}=0.12$} & \multicolumn{2}{|c|}{$V_{C N T}^{*}=0.17$} & \multicolumn{2}{|c|}{$V_{C N T}^{*}=0.28$} \\
\hline & & UD & FG-X & UD & FG-X & UD & FG-X \\
\hline \multirow[t]{2}{*}{1} & Shen [25] & 42.68 & 49.48 & 70.06 & 82.76 & 84.34 & 109.42 \\
\hline & Present & 42.83 & 49.11 & 70.28 & 82.26 & 84.67 & 109.67 \\
\hline \multirow[t]{2}{*}{$\sqrt{3}$} & Shen [25] & 23.40 & 25.60 & 38.98 & 43.83 & 45.11 & 55.84 \\
\hline & Present & 23.45 & 25.53 & 39.06 & 43.76 & 45.22 & 56.35 \\
\hline \multirow[t]{2}{*}{$\sqrt{5}$} & Shen [25] & 18.48 & 19.91 & 30.81 & 34.15 & 35.56 & 43.24 \\
\hline & Present & 18.51 & 19.88 & 30.85 & 34.11 & 35.62 & 43.65 \\
\hline
\end{tabular}

Buckling mode $(m, n)=(1,10)$ for $L / R=1,(m, n)=(1,8)$ for $L / R=\sqrt{3}$ and $(m, n)=(1,7)$ for $L / R=\sqrt{5}$.

The remainder of this section presents numerical results for buckling and postbuckling analyses for CNTRC TSSs under external pressure. Tab. 2 shows the effects of CNT volume fraction, distribution types and ratio of principal radii $R / a$ on the critical buckling pressures of CNTRC TSSs.

It is obvious that critical buckling loads are considerably increased as CNT volume fraction $V_{C N T}^{*}$ and/or $R / a$ ratio increase. For the most part, FG-X and FG-O types of CNT distribution give the highest and lowest critical buckling pressures, respectively, and UD type gives average value of critical buckling pressure. Particularly, for small values of $V_{C N T}^{*}$ and $R / a$ (for example $V_{C N T}^{*}=0.12$ and $R / a=0.1$ ), buckling pressure of 
Table 2. Effects of CNT volume fraction, distribution types and $R / a$ ratio on critical buckling pressures $q_{c r}(\mathrm{kPa})$ of CNTRC TSSs $\left(L / R=2, R / h=100, K_{1}=K_{2}=0\right)$

\begin{tabular}{ccccccc}
\hline$V_{C N T}^{*}$ & $R / a$ & UD & FG-X & FG-O & FG-V & FG- $\Lambda$ \\
\hline \multirow{2}{*}{0.12} & 0.1 & $61.82(1,10)$ & $64.23(1,10)$ & $57.16(2,11)$ & $64.33(2,11)$ & $57.07(2,11)$ \\
& 0.2 & $93.41(2,12)$ & $99.60(2,12)$ & $88.36(2,12)$ & $97.45(2,12)$ & $86.72(2,12)$ \\
& 0.5 & $192.19(3,15)$ & $206.65(3,16)$ & $173.03(4,16)$ & $199.38(3,15)$ & $174.46(4,16)$ \\
0.17 & 0.1 & $101.92(1,10)$ & $108.21(1,10)$ & $93.91(2,11)$ & $106.44(1,10)$ & $95.69(2,11)$ \\
& 0.2 & $155.00(2,12)$ & $168.37(2,12)$ & $145.92(2,12)$ & $162.45(2,12)$ & $145.68(2,12)$ \\
& 0.5 & $318.02(3,15)$ & $347.07(3,15)$ & $282.43(4,16)$ & $328.85(4,16)$ & $288.34(4,16)$ \\
0.28 & 0.1 & $120.69(1,10)$ & $137.60(1,9)$ & $106.96(2,11)$ & $126.66(2,11)$ & $116.61(2,11)$ \\
& 0.2 & $181.08(2,12)$ & $213.12(2,12)$ & $168.32(2,12)$ & $192.03(2,12)$ & $177.19(2,12)$ \\
& 0.5 & $374.33(3,16)$ & $440.43(3,15)$ & $331.82(4,17)$ & $393.32(3,15)$ & $360.21(3,15)$ \\
\hline
\end{tabular}

Numbers in parentheses indicate buckling mode $(m, n)$.

FG-V TSS approximately equals to that of FG-X TSS. Furthermore, numbers of waves in the buckling mode $(m, n)$ are increased when $R / a$ ratio becomes larger. Next, effects of geometrical ratios $L / R, R / h$ and surrounding elastic media on critical buckling pressures of FG-CNTRC TSSs are given in Tab. 3.

Table 3. Effects of geometrical ratios and elastic foundations on critical buckling pressures $q_{c r}(\mathrm{kPa})$ of CNTRC TSSs (FG-X, $\left.V_{C N T}^{*}=0.17, R / a=0.2\right)$

\begin{tabular}{cccccc}
\hline \multirow{2}{*}{$L / R$} & $\left(K_{1}, K_{2}\right)$ & \multicolumn{4}{c}{$R / h$} \\
\cline { 3 - 6 } & & 60 & 80 & 100 & 120 \\
\hline 1 & $(0,0)$ & $502.98(1,10)$ & $266.63(1,11)$ & $168.37(1,12)$ & $117.86(1,13)$ \\
& $(300,0)$ & $560.26(1,10)$ & $285.31(1,11)$ & $176.20(1,12)$ & $121.68(1,13)$ \\
& $(300,3)$ & $600.01(1,10)$ & $301.52(1,11)$ & $184.34(1,12)$ & $126.33(1,13)$ \\
1.5 & $(0,0)$ & $477.32(1,9)$ & $283.51(1,10)$ & $184.74(2,13)$ & $122.08(2,14)$ \\
& $(300,0)$ & $541.56(1,10)$ & $303.50(1,11)$ & $192.04(2,13)$ & $125.59(2,14)$ \\
& $(300,3)$ & $579.32(1,10)$ & $319.04(1,11)$ & $200.44(2,13)$ & $130.37(2,14)$ \\
2 & $(0,0)$ & $502.98(2,10)$ & $266.63(2,11)$ & $168.37(2,12)$ & $117.86(2,13)$ \\
& $(300,0)$ & $560.26(2,10)$ & $285.31(2,11)$ & $176.20(2,12)$ & $121.38(2,13)$ \\
& $(300,3)$ & $600.01(2,10)$ & $301.52(2,11)$ & $184.34(2,12)$ & $126.33(2,13)$ \\
\hline
\end{tabular}

Numbers in parentheses indicate buckling mode $(m, n)$.

As can be seen, critical buckling pressures are significantly enhanced as CNTRC TSSs are surrounded by elastic foundations, especially Pasternak type foundations. It is noted that buckling pressure is decreased when $L / R$ increases from 1 to 1.5 for thicker TSSs (i.e. $R / h=60$ ) and buckling pressure is increased when $L / R$ increases from 1 to 1.5 for thinner TSSs (i.e. $R / h \geq 80$ ). 
In what follows, postbuckling behavior of CNTRC TSSs will be analyzed graphically. Fig. 2 considers the effects of CNT distribution types on the postbuckling behavior of CNTRC TSSs under external pressure. It is clear that FG-X and FG-O TSSs have the strongest and weakest postbuckling strengths, respectively. In addition, FG-V type of CNT distribution gives relatively high equilibrium path in small region of postbuckling response. In general, CNTRC TSS with UD type has only average level of postbuckling load carrying capacity. Subsequently, the effects of volume fraction $V_{C N T}^{*}$ of carbon nanotubes on the postbuckling behavior of CNTRC TSSs are analyzed in Fig. 3. As can be observed, buckling load and postbuckling curve are rapidly enhanced as $V_{C N T}^{*}$ increases from 0.12 to 0.17 , then, these are slowly increased when $V_{C N T}^{*}$ varies from 0.17 to 0.28 . Furthermore, it is noted from Fig. 3 that intensity of snap-through response (i.e. difference between bifurcation value and the lowest value on pressure-deflection curve) becomes more severe with higher values of CNT volume percentage.

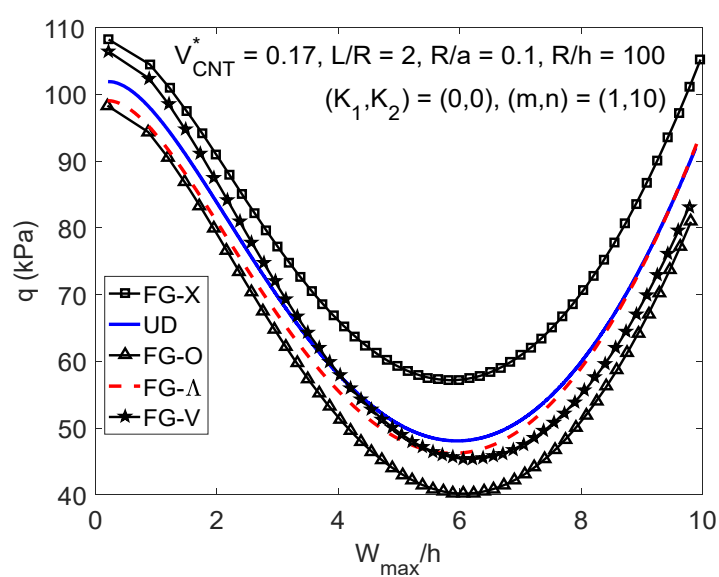

Fig. 2. Effects of CNT distribution types on postbuckling behavior of CNTRC toroidal shell segments under uniform external pressure

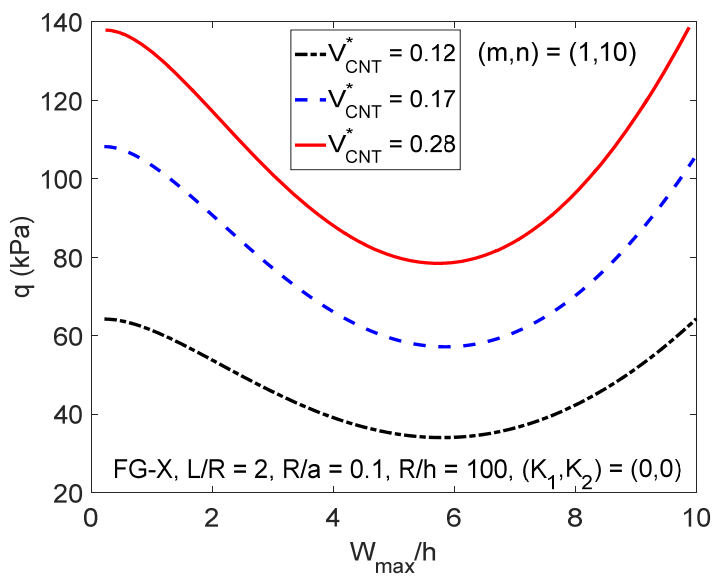

Fig. 3. Effects of CNT volume fractions on postbuckling behavior of CNTRC toroidal shell segments under uniform external pressure

The effects of $R / a$ ratio and foundation stiffness on the postbuckling behavior of CNTRC TSSs under external pressure are considered in Figs. 4 and 5, respectively. Fig. 4 indicates that buckling pressure is smaller and postbuckling pressure-deflection response is more stable (i.e. more benign snap-through phenomenon) when CNTRC TSS is more shallow (i.e. smaller value of $R / a$ ratio). In contrast, buckling pressure is remarkably higher and snap-through instability is more severe as $R / a$ ratio become larger. It is interesting that, although CNTRC cylindrical shell $(R / a=0)$ is buckled sooner, its load carrying capacity in the deep region of postbuckling response is better.

Finally, it is recognized from Fig. 5 that buckling pressure and pressure-deflection curves of CNTRC TSSs are pronouncedly enhanced due to surrounding elastic media. Specifically, load carrying capacity of CNTRC TSSs are considerably improved as nondimensional stiffness parameters $K_{1}$ and $K_{2}$ are increased, although intensity of snapthrough response is almost not reduced. 


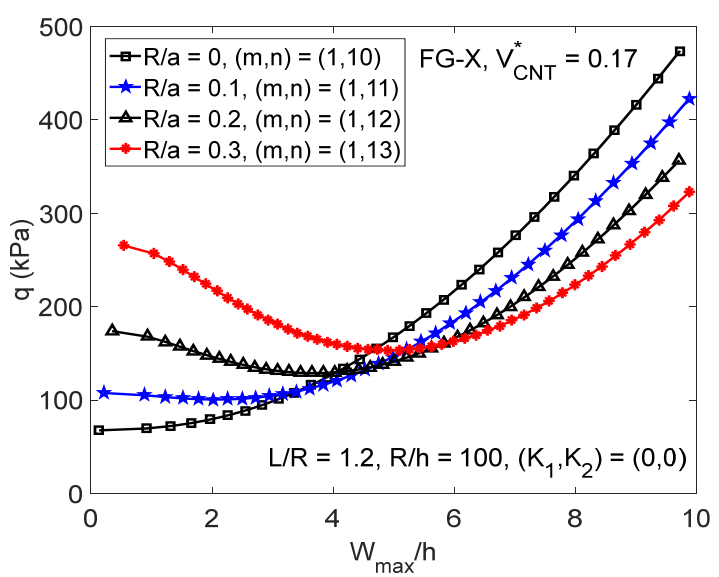

Fig. 4. Effects of geometrical ratio $R / a$ on postbuckling behavior of CNTRC toroidal shell segments under uniform external pressure

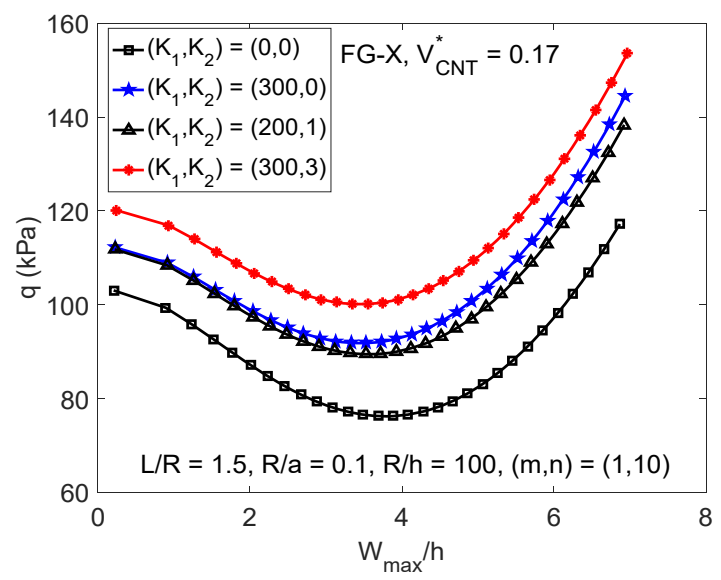

Fig. 5. Effects of elastic foundations on postbuckling behavior of CNTRC toroidal shell segments under uniform external pressure

\section{CONCLUDING REMARKS}

Buckling and postbuckling behaviors of CNTRC toroidal shell segments surrounded by elastic media and subjected to uniform external pressure have been presented in this paper. An analytical approach with three-term solution of deflection and Galerkin method are used for formulation. From above analysis, the following remarks are reached:

1. CNT distribution has significant influence on buckling and postbuckling behaviors of CNTRC TSSs. In general, FG-X type of CNT distribution gives the highest buckling pressure and the strongest postbuckling strength of CNTRC TSSs. In particular, for shallow and CNT-poor TSS (i.e. smaller values of $R / a$ and $V_{C N T}^{*}$ ), FG-V type of CNT distribution gives relatively high buckling load and load-deflection curve.

2. Buckling load and load-deflection curve are rapidly enhanced when CNT volume fraction increases from 0.12 to 0.17 and then these are slowly increased as CNT volume fraction varies from 0.17 to 0.28 . However, increase in CNT volume percentage results in more intense snap-through response.

3. Critical buckling pressure of CNTRC TSSs is considerably enhanced as $R / a$ ratio is increased. However, this increase in buckling pressure is accompanied by a relatively severe snap-through instability in the postbuckling region.

4. Buckling load and postbuckling load carrying capability of pressure-loaded CNTRC TSSs are pronouncedly improved due to surrounding elastic foundations, although intensity of snap-through response is almost unchanged.

\section{ACKNOWLEDGMENT}

This research is funded by Vietnam National Foundation for Science and Technology Development (NAFOSTED) under grant number 107.02-2017.11. 


\section{REFERENCES}

[1] E. T. Thostenson, C. Li, and T. W. Chou. Nanocomposites in context. Composites Science and Technology, 65, (3-4), (2005), pp. 491-516. https://doi.org/10.1016/j.compscitech.2004.11.003.

[2] J. N. Coleman, U. Khan, W. J. Blau, and Y. K. Gunko. Small but strong: a review of the mechanical properties of carbon nanotube-polymer composites. Carbon, 44, (9), (2006), pp. 16241652. https://doi.org/10.1016/j.carbon.2006.02.038.

[3] Z. Spitalsky, D. Tasis, K. Papagelis, and C. Galiotis. Carbon nanotube-polymer composites: chemistry, processing, mechanical and electrical properties. Progress in Polymer Science, 35, (3), (2010), pp. 357-401. https://doi.org/10.1016/j.progpolymsci.2009.09.003.

[4] H. S. Shen. Nonlinear bending of functionally graded carbon nanotube-reinforced composite plates in thermal environments. Composite Structures, 91, (1), (2009), pp. 9-19. https://doi.org/10.1016/j.compstruct.2009.04.026.

[5] Z. X. Lei, K. M. Liew, and J. L. Yu. Buckling analysis of functionally graded carbon nanotubereinforced composite plates using the element-free kp-Ritz method. Composite Structures, 98, (2013), pp. 160-168. https://doi.org/10.1016/j.compstruct.2012.11.006.

[6] L. W. Zhang, Z. X. Lei, and K. M. Liew. Buckling analysis of FG-CNT reinforced composite thick skew plates using an element-free approach. Composites Part B: Engineering, 75, (2015), pp. 36-46. https://doi.org/10.1016/j.compositesb.2015.01.033.

[7] M. Wang, Z. M. Li, and P. Qiao. Semi-analytical solutions to buckling and free vibration analysis of carbon nanotube-reinforced composite thin plates. Composite Structures, 144, (2016), pp. 33-43. https://doi.org/10.1016/j.compstruct.2016.02.025.

[8] N. Wattanasakulpong and A. Chaikittiratana. Exact solutions for static and dynamic analyses of carbon nanotube-reinforced composite plates with pasternak elastic foundation. Applied Mathematical Modelling, 39, (18), (2015), pp. 5459-5472. https://doi.org/10.1016/j.apm.2014.12.058.

[9] M. Mirzaei and Y. Kiani. Thermal buckling of temperature dependent FG-CNT reinforced composite plates. Meccanica, 51, (9), (2016), pp. 2185-2201. https://doi.org/10.1007/s11012015-0348-0.

[10] Y. Kiani. Thermal buckling of temperature-dependent FG-CNT-reinforced composite skew plates. Journal of Thermal Stresses, 40, (11), (2017), pp. 1442-1460. https://doi.org/10.1080/01495739.2017.1336742.

[11] P. Malekzadeh and M. Shojaee. Buckling analysis of quadrilateral laminated plates with carbon nanotubes reinforced composite layers. Thin-Walled Structures, 71, (2013), pp. 108-118. https://doi.org/10.1016/j.tws.2013.05.008.

[12] A. R. Setoodeh and M. Shojaee. Critical buckling load optimization of functionally graded carbon nanotube-reinforced laminated composite quadrilateral plates. Polymer Composites, 39, (S2), (2018), pp. E853-E868. https://doi.org/10.1002/pc.24289.

[13] H. S. Shen and C. L. Zhang. Thermal buckling and postbuckling behavior of functionally graded carbon nanotube-reinforced composite plates. Materials \& Design, 31, (7), (2010), pp. 3403-3411. https://doi.org/10.1016/j.matdes.2010.01.048.

[14] Y. Kiani. Thermal post-buckling of FG-CNT reinforced composite plates. Composite Structures, 159, (2017), pp. 299-306. https://doi.org/10.1016/j.compstruct.2016.09.084.

[15] L. W. Zhang and K. M. Liew. Postbuckling analysis of axially compressed CNT reinforced functionally graded composite plates resting on Pasternak foundations using an element-free approach. Composite Structures, 138, (2016), pp. 40-51. https://doi.org/10.1016/j.compstruct.2015.11.031. 
[16] H. V. Tung. Thermal buckling and postbuckling behavior of functionally graded carbon-nanotube-reinforced composite plates resting on elastic foundations with tangential-edge restraints. Journal of Thermal Stresses, 40, (5), (2017), pp. 641-663. https://doi.org/10.1080/01495739.2016.1254577.

[17] L. T. N. Trang and H. V. Tung. Tangential edge constraint sensitivity of nonlinear stability of CNT-reinforced composite plates under compressive and thermomechanical loadings. Journal of Engineering Mechanics, 144, (7), (2018). https://doi.org/10.1061/(asce)em.19437889.0001479 .

[18] M. Nasihatgozar, V. Daghigh, M. Eskandari, K. Nikbin, and A. Simoneau. Buckling analysis of piezoelectric cylindrical composite panels reinforced with carbon nanotubes. International Journal of Mechanical Sciences, 107, (2016), pp. 69-79. https://doi.org/10.1016/j.ijmecsci.2016.01.010.

[19] E. García-Macías, L. Rodriguez-Tembleque, R. Castro-Triguero, and A. Sáez. Buckling analysis of functionally graded carbon nanotube-reinforced curved panels under axial compression and shear. Composites Part B: Engineering, 108, (2017), pp. 243-256. https://doi.org/10.1016/j.compositesb.2016.10.002.

[20] H. S. Shen and Y. Xiang. Postbuckling of axially compressed nanotube-reinforced composite cylindrical panels resting on elastic foundations in thermal environments. Composites Part $B$ : Engineering, 67, (2014), pp. 50-61. https://doi.org/10.1016/j.compositesb.2014.06.020.

[21] H.S. Shen. Postbuckling of nanotube-reinforced composite cylindrical panels resting on elastic foundations subjected to lateral pressure in thermal environments. Engineering Structures, 122, (2016), pp. 174-183. https://doi.org/10.1016/j.engstruct.2016.05.004.

[22] L. T. N. Trang and H. V. Tung. Thermomechanical nonlinear analysis of axially compressed carbon nanotube-reinforced composite cylindrical panels resting on elastic foundations with tangentially restrained edges. Journal of Thermal Stresses, 41, (4), (2018), pp. 418-438. https://doi.org/10.1080/01495739.2017.1409093.

[23] H. V. Tung and L. T. N. Trang. Imperfection and tangential edge constraint sensitivities of thermomechanical nonlinear response of pressure-loaded carbon nanotubereinforced composite cylindrical panels. Acta Mechanica, 229, (5), (2018), pp. 1949-1969. https://doi.org/10.1007/s00707-017-2093-z.

[24] H. S. Shen. Postbuckling of nanotube-reinforced composite cylindrical shells in thermal environments, Part I: Axially-loaded shells. Composite Structures, 93, (8), (2011), pp. 2096-2108. https://doi.org/10.1016/j.compstruct.2011.02.011.

[25] H. S. Shen. Postbuckling of nanotube-reinforced composite cylindrical shells in thermal environments, Part II: Pressure-loaded shells. Composite Structures, 93, (10), (2011), pp. 2496-2503. https://doi.org/10.1016/j.compstruct.2011.04.005.

[26] H. S. Shen. Thermal buckling and postbuckling behavior of functionally graded carbon nanotube-reinforced composite cylindrical shells. Composites Part B: Engineering, 43, (3), (2012), pp. 1030-1038. https://doi.org/10.1016/j.compositesb.2011.10.004.

[27] H. S. Shen. Torsional postbuckling of nanotube-reinforced composite cylindrical shells in thermal environments. Composite Structures, 116, (2014), pp. 477-488. https://doi.org/10.1016/j.compstruct.2014.05.039.

[28] R. Ansari, T. Pourashraf, R. Gholami, and A. Shahabodini. Analytical solution for nonlinear postbuckling of functionally graded carbon nanotube-reinforced composite shells with piezoelectric layers. Composites Part B: Engineering, 90, (2016), pp. 267-277. https://doi.org/10.1016/j.compositesb.2015.12.012. 
[29] D. G. Ninh. Nonlinear thermal torsional post-buckling of carbon nanotube-reinforced composite cylindrical shell with piezoelectric actuator layers surrounded by elastic medium. Thin-Walled Structures, 123, (2018), pp. 528-538. https://doi.org/10.1016/j.tws.2017.11.027.

[30] B. H. Dao, N. G. Dinh, and T. I. Tran. Buckling analysis of eccentrically stiffened functionally graded toroidal shell segments under mechanical load. Journal of Engineering Mechanics, 142, (1), (2015). https://doi.org/10.1061/(asce)em.1943-7889.0000964.

[31] D. H. Bich and D. G. Ninh. Post-buckling of sigmoid-functionally graded material toroidal shell segment surrounded by an elastic foundation under thermo-mechanical loads. Composite Structures, 138, (2016), pp. 253-263. https://doi.org/10.1016/j.compstruct.2015.11.044.

[32] O. Gohardani, M. C. Elola, and C. Elizetxea. Potential and prospective implementation of carbon nanotubes on next generation aircraft and space vehicles: A review of current and expected applications in aerospace sciences. Progress in Aerospace Sciences, 70, (2014), pp. 42 68. https://doi.org/10.1016/j.paerosci.2014.05.002.

[33] D. O. Brush and B. O. Almroth. Buckling of bars, plates, and shells. McGraw-Hill, New York, (1975).

[34] D. Van Dung and L. K. Hoa. Solving nonlinear stability problem of imperfect functionally graded circular cylindrical shells under axial compression by Galerkin's method. Vietnam Journal of Mechanics, 34, (3), (2012), pp. 139-156. https://doi.org/10.15625/0866$7136 / 34 / 3 / 2356$.

[35] L. T. N. Trang and H. V. Tung. Buckling and postbuckling of carbon nanotube-reinforced composite cylindrical panels subjected to axial compression in thermal environments. Vietnam Journal of Mechanics, 40, (1), (2018), pp. 47-61.

\section{APPENDIX A}

The coefficients $a_{j 3}(j=1 \div 4)$ and $a_{k 4}(k=1 \div 3)$ in the Eqs. (20b) and (20c) are

$$
\begin{aligned}
& a_{13}=a_{11} \beta_{m}^{4}+a_{21} \delta_{n}^{4}+a_{31} \beta_{m}^{2} \delta_{n}^{2}+k_{1}+k_{2}\left(\beta_{m}^{2}+\delta_{n}^{2}\right) \\
& +\frac{1}{a_{12} \beta_{m}^{4}+a_{22} \beta_{m}^{2} \delta_{n}^{2}+a_{32} \delta_{n}^{4}}\left(\frac{\beta_{m}^{2}}{R}+\frac{\delta_{n}^{2}}{a}-a_{42} \beta_{m}^{4}-a_{52} \beta_{m}^{2} \delta_{n}^{2}-a_{62} \delta_{n}^{4}\right)\left(a_{41} \beta_{m}^{2} \delta_{n}^{2}+\frac{\beta_{m}^{2}}{R}+\frac{\delta_{n}^{2}}{a}\right), \\
& a_{23}=\frac{\beta_{m}^{2} \delta_{n}^{2}}{a_{12} \beta_{m}^{4}+a_{22} \beta_{m}^{2} \delta_{n}^{2}+a_{32} \delta_{n}^{4}}\left[2 \frac{\beta_{m}^{2}}{R}+2 \frac{\delta_{n}^{2}}{a}+\left(a_{41}-a_{52}\right) \beta_{m}^{2} \delta_{n}^{2}-a_{42} \beta_{m}^{4}-a_{62} \delta_{n}^{4}\right] \\
& +\frac{\delta_{n}^{2}}{16 a_{12}}\left(\frac{4}{R}-16 a_{42} \beta_{m}^{2}\right), \\
& a_{33}=\frac{\beta_{m}^{4}}{16 a_{32}}+\frac{\delta_{n}^{4}}{16 a_{12}}, \\
& a_{43}=\frac{\beta_{m}^{4} \delta_{n}^{4}}{a_{12} \beta_{m}^{4}+a_{22} \beta_{m}^{2} \delta_{n}^{2}+a_{32} \delta_{n}^{4}}+\frac{\beta_{m}^{4} \delta_{n}^{4}}{81 a_{12} \beta_{m}^{4}+9 a_{22} \beta_{m}^{2} \delta_{n}^{2}+a_{32} \delta_{n}^{4}}, \\
& a_{14}=4 a_{11} \beta_{m}^{4}+\frac{3}{4} k_{1}+\beta_{m}^{2} k_{2}-\frac{1}{4 a_{12} R}\left(4 a_{42} \beta_{m}^{2}-\frac{1}{R}\right), \\
& a_{24}=\frac{\beta_{m}^{2} \delta_{n}^{2}}{2\left(a_{12} \beta_{m}^{4}+a_{22} \beta_{m}^{2} \delta_{n}^{2}+a_{32} \delta_{n}^{4}\right)}\left(\frac{\beta_{m}^{2}}{R}+\frac{\delta_{n}^{2}}{a}-a_{42} \beta_{m}^{4}-a_{52} \beta_{m}^{2} \delta_{n}^{2}-a_{62} \delta_{n}^{4}\right)+\frac{\delta_{n}^{2}}{16 a_{12} R}, \\
& a_{34}=\frac{\beta_{m}^{4} \delta_{n}^{4}}{2\left(a_{12} \beta_{m}^{4}+a_{22} \beta_{m}^{2} \delta_{n}^{2}+a_{32} \delta_{n}^{4}\right)}+\frac{\beta_{m}^{4} \delta_{n}^{4}}{2\left(81 a_{12} \beta_{m}^{4}+9 a_{22} \beta_{m}^{2} \delta_{n}^{2}+a_{32} \delta_{n}^{4}\right)} .
\end{aligned}
$$


The details of coefficients $b_{i 1}(i=1 \div 2), b_{j 2}(j=1 \div 5)$ and $b_{k 3}(k=1 \div 4)$ in the Eqs. (23)-(25) are defined as follows

$$
\begin{aligned}
& b_{11}=\frac{1}{2 R_{h}^{2}}\left(1-v_{12} v_{21}\right) \bar{e}_{21}+\frac{K_{1} E^{m}}{2 R_{h}^{4}}, \quad b_{21}=\frac{n^{2}}{8 R_{h}^{3}}\left(1-v_{12} v_{21}\right) \bar{e}_{21}, \\
& b_{12}=\bar{a}_{11} \frac{m^{4} \pi^{4}}{R_{h}^{4} L_{R}^{4}}+\bar{a}_{21} \frac{n^{4}}{R_{h}^{4}}+\bar{a}_{31} \frac{m^{2} n^{2} \pi^{2}}{R_{h}^{4} L_{R}^{2}}+K_{1} \frac{E^{m}}{R_{h}^{4}}+K_{2} \frac{E^{m}}{R_{h}^{2}}\left(\frac{m^{2} \pi^{2}}{R_{h}^{2} L_{R}^{2}}+\frac{n^{2}}{R_{h}^{2}}\right) \\
& +\left(\frac{m^{2} \pi^{2}}{R_{h}^{3} L_{R}^{2}}+\frac{n^{2} R_{a}}{R_{h}^{3}}-\bar{a}_{42} \frac{m^{4} \pi^{4}}{R_{h}^{4} L_{R}^{4}}-\bar{a}_{52} \frac{m^{2} n^{2} \pi^{2}}{R_{h}^{4} L_{R}^{2}}-\bar{a}_{62} \frac{n^{4}}{R_{h}^{4}}\right)\left(\frac{\bar{a}_{41} m^{2} n^{2} \pi^{2} L_{R}^{2}+m^{2} \pi^{2} R_{h} L_{R}^{2}+n^{2} R_{h} L_{R}^{4} R_{a}}{\bar{a}_{12} m^{4} \pi^{4}+\bar{a}_{22} m^{2} n^{2} \pi^{2} L_{R}^{2}+\bar{a}_{32} n^{4} L_{R}^{4}}\right), \\
& b_{22}=\frac{n^{2}}{R_{h}^{3}} \bar{e}_{21}\left(1-v_{12} v_{21}\right) \text {, } \\
& b_{32}=\frac{1}{R_{h}^{4}\left(\bar{a}_{12} m^{4} \pi^{4}+\bar{a}_{22} m^{2} n^{2} \pi^{2} L_{R}^{2}+\bar{a}_{32} n^{4} L_{R}^{4}\right)}\left[\bar{a}_{41} m^{4} n^{4} \pi^{4}+m^{4} n^{2} \pi^{4} R_{h}+m^{2} n^{4} \pi^{2} L_{R}^{2} R_{h} R_{a}\right. \\
& \left.+m^{2} n^{2} \pi^{2} R_{h}^{4} L_{R}^{2}\left(\frac{m^{2} \pi^{2}}{R_{h}^{3} L_{R}^{2}}+\frac{n^{2} R_{a}}{R_{h}^{3}}-\bar{a}_{42} \frac{m^{4} \pi^{4}}{R_{h}^{4} L_{R}^{4}}-\bar{a}_{52} \frac{m^{2} n^{2} \pi^{2}}{R_{h}^{4} L_{R}^{2}}-\bar{a}_{62} \frac{n^{4}}{R_{h}^{4}}\right)\right] \\
& +\frac{n^{2}}{16 \bar{a}_{12} R_{h}^{2}}\left(\frac{4}{R_{h}}-16 \bar{a}_{42} \frac{m^{2} \pi^{2}}{R_{h}^{2} L_{R}^{2}}\right)+\frac{n^{2} \bar{e}_{21}}{2 R_{h}^{3}}\left(1-v_{12} v_{21}\right), \\
& b_{42}=\frac{m^{4} \pi^{4}}{16 \bar{a}_{32} R_{h}^{4} L_{R}^{4}}+\frac{n^{4}}{16 \bar{a}_{12} R_{h}^{4}}+\frac{n^{4} \bar{e}_{21}}{8 R_{h}^{4}}\left(1-v_{12} v_{21}\right) \text {, } \\
& b_{52}=\frac{m^{4} n^{4} \pi^{4}}{R_{h}^{4}\left(\bar{a}_{12} m^{4} \pi^{4}+\bar{a}_{22} m^{2} n^{2} \pi^{2} L_{R}^{2}+\bar{a}_{32} n^{4} L_{R}^{4}\right)}+\frac{m^{4} n^{4} \pi^{4}}{R_{h}^{4}\left(81 \bar{a}_{12} m^{4} \pi^{4}+9 \bar{a}_{22} m^{2} n^{2} \pi^{2} L_{R}^{2}+\bar{a}_{32} n^{4} L_{R}^{4}\right)}, \\
& b_{13}=K_{1} \frac{E^{m}}{R_{h}^{4}}+\frac{\bar{e}_{21}}{R_{h}^{2}}\left(1-v_{12} v_{21}\right) \text {, } \\
& b_{23}=\frac{\bar{e}_{21}}{2 R_{h}^{2}}\left(1-v_{12} v_{21}\right)+4 \bar{a}_{11} \frac{m^{4} \pi^{4}}{R_{h}^{4} L_{R}^{4}}+K_{1} \frac{3 E^{m}}{4 R_{h}^{4}}+K_{2} \frac{m^{2} \pi^{2} E^{m}}{R_{h}^{4} L_{R}^{2}}-\frac{1}{4 R_{h} \bar{a}_{12}}\left(4 \bar{a}_{42} \frac{m^{2} \pi^{2}}{R_{h}^{2} L_{R}^{2}}-\frac{1}{R_{h}}\right), \\
& b_{33}=\frac{n^{2} \bar{e}_{21}}{8 R_{h}^{3}}\left(1-v_{12} v_{21}\right)+\frac{n^{2}}{16 \bar{a}_{12} R_{h}^{3}} \\
& +\frac{m^{2} n^{2} \pi^{2} L_{R}^{2}}{2\left(\bar{a}_{12} m^{4} \pi^{4}+\bar{a}_{22} m^{2} n^{2} \pi^{2} L_{R}^{2}+\bar{a}_{32} n^{4} L_{R}^{4}\right)}\left(\frac{m^{2} \pi^{2}}{R_{h}^{3} L_{R}^{2}}+\frac{n^{2} R_{a}}{R_{h}^{3}}-\bar{a}_{42} \frac{m^{4} \pi^{4}}{R_{h}^{4} L_{R}^{4}}-\bar{a}_{52} \frac{m^{2} n^{2} \pi^{2}}{R_{h}^{4} L_{R}^{2}}-\bar{a}_{62} \frac{n^{4}}{R_{h}^{4}}\right), \\
& b_{43}=\frac{m^{4} n^{4} \pi^{4}}{2 R_{h}^{4}\left(\bar{a}_{12} m^{4} \pi^{4}+\bar{a}_{22} m^{2} n^{2} \pi^{2} L_{R}^{2}+\bar{a}_{32} n^{4} L_{R}^{4}\right)}+\frac{m^{4} n^{4} \pi^{4}}{2 R_{h}^{4}\left(81 \bar{a}_{12} m^{4} \pi^{4}+9 \bar{a}_{22} m^{2} n^{2} \pi^{2} L_{R}^{2}+\bar{a}_{32} n^{4} L_{R}^{4}\right)},
\end{aligned}
$$

in which

$$
\begin{aligned}
& \left(\bar{a}_{11}, \bar{a}_{21}, \bar{a}_{31}, \bar{e}_{13}, \bar{e}_{23}, \bar{e}_{33}\right)=\frac{1}{h^{3}}\left(a_{11}, a_{21}, a_{31}, e_{13}, e_{23}, e_{33}\right), \quad\left(\bar{a}_{12}, \bar{a}_{22}, \bar{a}_{32}\right)=h\left(a_{12}, a_{22}, a_{32}\right), \\
& \left(\bar{a}_{41}, \bar{a}_{42}, \bar{a}_{52}, \bar{a}_{62}, \bar{e}_{11}, \bar{e}_{21}, \bar{e}_{31}\right)=\frac{1}{h}\left(a_{41}, a_{42}, a_{52}, a_{62}, e_{11}, e_{21}, e_{31}\right),
\end{aligned}
$$




$$
\begin{aligned}
& \left(\bar{e}_{12}, \bar{e}_{22}, \bar{e}_{32}\right)=\frac{1}{h^{2}}\left(e_{12}, e_{22}, e_{32}\right), \\
& R_{h}=R / h, \quad R_{a}=R / a, \quad L_{R}=L / R, \quad\left(K_{1}, K_{2}\right)=\left(k_{1} R^{2}, k_{2}\right) \frac{R^{2}}{E^{m} h^{3}} .
\end{aligned}
$$

\section{APPENDIX B}

The details of the coefficients $b_{i 4}$ and $b_{i 5}(i=1 \div 4)$ in Eqs. (27a) and (27b) are defined as follows

$$
\begin{aligned}
& \left(b_{14}, b_{24}, b_{34}, b_{44}\right)=\frac{1}{b_{54}}\left(b_{12} b_{21}, b_{11} b_{42}-b_{21} b_{32}, b_{21} b_{52}, b_{42}\right) \\
& \left(b_{15}, b_{25}, b_{35}, b_{45}\right)=\frac{1}{b_{55}}\left(2 b_{11} b_{12}, b_{11} b_{22}-2 b_{11} b_{32}, 2 b_{11} b_{52}, b_{22}\right) \\
& b_{54}=b_{55}=b_{21} b_{22}-2 b_{11} b_{42} .
\end{aligned}
$$

The coefficients $b_{i 6}(i=1 \div 6)$ in Eq. (28) are determined as follows

$$
\begin{aligned}
& b_{16}=1+b_{13} b_{44}-b_{33} b_{45}, \quad b_{26}=b_{43} b_{45}, \quad b_{36}=b_{13} b_{14}-b_{33} b_{15}, \\
& b_{46}=b_{13} b_{24}+b_{23}-b_{33} b_{25}+b_{15} b_{43}, \quad b_{56}=b_{13} b_{34}+b_{25} b_{43}-b_{33} b_{35}, \quad b_{66}=b_{35} b_{43} .
\end{aligned}
$$

